\title{
山西芦芽山14种常见灌木生物量模型及生物量分配
}

\author{
罗永开 ${ }^{1,2}$ 方精云 ${ }^{1}$ 胡会峰 $^{*}$ \\ ${ }^{1}$ 中国科学院植物研究所植被与环境变化国家重点实验室, 北京 100093 ; ${ }^{2}$ 中国科学院大学, 北京 100049
}

摘 要 灌木生物量模型是估算灌木生物量的重要方法, 而灌木生物量在各器官间的分配是其适应周围环境的重要体现。基 于对山西芦芽山地区14种常见灌木的各器官(根、茎和叶)、地上和总生物量, 以及基径、树高、冠幅的测定, 建立了各器官、 地上及总生物量的最优估算模型, 探究了各器官生物量与总生物量(如叶质比、茎质比及根质比)及地上-地下生物量(根冠比) 的关系。结果表明: (1)总体而言, 幂函数和线性函数对这些灌木生物量的估测效果较好。(2)生长低矮、分枝数多的灌木种采 用冠幅面积估测生物量效果较好; 生长直立或分枝数少的灌木种采用总基径的平方与茎干高度乘积估测生物量效果较好; 其他介于两者之间的灌木种采用冠幅体积估测生物量效果较好。(3) 14 种灌木的平均根冠比是 0.61 , 叶质比 0.17 , 茎质比 0.48 , 根质比 0.35 ; 此外, 带刺灌木种除叶质比显著大于不带刺灌木种外, 茎质比、根质比和根冠比都显著小于不带刺灌木种。

关键词 灌木; 生物量; 芦芽山; 叶质比; 根冠比

引用格式: 罗永开, 方精云, 胡会峰 (2017). 山西芦芽山14种常见灌木生物量模型及生物量分配. 植物生态学报, 41, 115-125. doi: 10.17521/cjpe.2016.0131

\section{Biomass estimation models and allocation patterns of 14 shrub species in Mountain Luya, Shanxi, China}

LUO Yong-Kai ${ }^{1,2}$, FANG Jing-Yun ${ }^{1}$, and HU Hui-Feng ${ }^{1 *}$

${ }^{1}$ State Key Laboratory of Vegetation and Environmental Change, Institute of Botany, Chinese Academy of Sciences, Beijing 100093, China; and ${ }^{2}$ University of Chinese Academy of Sciences, Beijing 100049, China

\section{Abstract}

Aims Shrub species have evolved specific strategies to regulate biomass allocation among various organs or between above- and belowground biomass and shrub biomass model is an important approach to estimate biomass allocation among different shrub species. This study was designed to establish the optimal estimation models for each organ (leaf, stem, and root), aboveground and total biomass of 14 common shrub species in Mountain Luya, Shanxi Province, China. Furthermore, we explored biomass allocation characteristics of these shrub species by using the index of leaf biomass fraction (leaf to total biomass), stem biomass fraction (stem to total biomass), root biomass fraction (root to total biomass), and root/shoot ratio (R/S) (belowground to aboveground biomass).

Methods We used plant height, basal diameter, canopy diameter and their combination as variables to establish the optimal biomass estimation models for each shrub species. In addition, we used the ratios of leaf, stem, root to total biomass, and belowground to aboveground biomass to explore the difference of biomass allocation patterns of 14 shrub species.

Important findings Most of biomass estimation models could be well expressed by the exponential and linear functions. Biomass for shorter shrub species with more stems could be better estimated by canopy area; biomass for taller shrub species with less stems could be better estimated by the sum of the square of total base diameter multiply stem height; and biomass for the rest shrub species could be better estimated by canopy volume. The averaged value for these shrub species was $0.61,0.17,0.48$, and 0.35 for $\mathrm{R} / \mathrm{S}$, leaf biomass fraction, stem biomass fraction, and root biomass fraction, respectively. Except for leaf biomass fraction, R/S, stem biomass fraction, and root biomass fraction for shrubs with thorn was significantly greater than that for shrubs without thorn.

Key words shrub; biomass; Mountain Luya; leaf biomass fraction; root/shoot ratio

Citation: Luo YK, Fang JY, Hu HF (2017). Biomass estimation models and allocation patterns of 14 shrub species in Mountain Luya, Shanxi, China. Chinese Journal of Plant Ecology, 41, 115-125. doi: 10.17521/cjpe.2016.0131

收稿日期Received: 2016-04-11 接受日期Accepted: 2016-07-23

* 通信作者Author for correspondence (E-mail: huifhu@ibcas.ac.cn) 
生物量是生态系统生产力的主要组成成分, 对 生态系统功能体现和群落结构形成都起着重要作用 (Bloom et al., 1985; 刘长成等, 2009)。灌木作为陆地 生态系统一种重要的生活型, 以较强的抗逆性及特 殊的形态特征在全球陆地广泛分布, 它不仅在保持 水土、防风固沙等方面有着不可替代的作用, 而且 是陆地生态系统物质循环和能量流动的重要组成部 分(俞海生等, 2003)。灌木生物量作为灌木群落和生 态系统研究的重要指标, 它不仅是灌木与乔木幼苗 竞争能力的反映, 而且反映了灌丛生态系统对周围 环境的适应。刏割法是研究灌木生物量的一种方法, 但这种方法费事费力, 破坏性较大。通过建立生物 量和灌木形态指标之间的关系来估测灌木的生物 量, 是现在常用的估算灌木生物量的方法。关于灌 木生物量模型, 国外如Whittaker (1982)对灌丛的净 生产力和生物量有过研究, Salis等(2006)对巴西稀 树草原灌木地上生物量建立过模型。国内较早见于 姜凤岐和卢凤勇(1982)对小叶锦鸡儿(Caragana microphylla) 等少数几种灌木的地上生物量建立过 生物量方程。之后, 关于灌木生物量模型的研究陆 续出现(陈遐林等, 2002; 张海清等, 2005; 蔡哲等, 2006; 方精云等, 2006; 曾慧卿等, 2006, 2007; 林伟 等, 2010)。通过数理分析得到的最佳生物量方程不 仅为灌木生物量的连续监测提供了可能, 而且可用 于对类似环境灌木生物量的估算(曾慧卿等, 2006)。

生物量分配是表征植物生理过程的重要指标 (Enquist \& Niklas, 2002), 主要受植物的遗传特性、 环境条件、个体大小、植物功能型的影响, 因而在 不同环境与生长压力下植物有不同的生长特性与物 质分配规律 (程栋梁, 2007; 李旭东等, 2012; Pooter et al., 2012)。

目前关于生物量分配的研究多集中于乔木，而 有关灌木生物量分配的研究较少。雷蕾(2012)对祁 连山高山灌丛生物量分配有过研究, 刘兴良等 (2006)探讨过川滇高山栋(Quercus aquifolioides)灌 丛地上生物量分配的特征。相比于乔木, 灌木所处 的环境往往较为干旱、贫痊。为适应所处环境, 灌 木往往会呈现带刺、根深等特殊的形态特征(易海 燕, 2010)。因此, 刺不仅是植物防御敌害的生存策 略, 而且是植物对干旱环境适应的一种形态特征。 而生长在较为干旱环境中的带刺与不带刺灌木之间 生物量分配是否有差异, 带刺灌木是否改变了生物
量分配策略的研究目前较为少见。

芦芽山国家自然保护区位于山西省忻州市, 保 护区内植被类型丰富, 森林覆盖率达到 $58.8 \%$, 灌 木种类较多, 但关于当地灌木生物量估测及生物量 分配方面的研究鲜有报道。为准确评估保护区内的 森林资源, 为保护区内灌丛生态系统生物量的持续 调查提供依据, 本研究通过调查保护区内14种常见 灌木的形态因子与生物量, 建立了保护区内 14 种常 见灌木的最优生物量估算模型; 此外, 还对这些灌 木种各器官间的生物量分配规律及带刺与不带刺灌 木种之间的生物量分配差异进行了探讨。这些研究 一方面为保护区估算灌木群落生物量提供了准确、 简洁的方法, 另一方面有助于完善对灌木生物量分 配规律的认识。

\section{1 材料和方法}

\section{1 研究区概况}

山西芦芽山国家级自然保护区位于山西省吕梁 山脉北端, 地处宁武、五寨、岢岗三县的交界处, $111.83^{\circ}-112.09^{\circ} \mathrm{E}, 38.59^{\circ}-38.75^{\circ} \mathrm{N}$, 区内最高海拔 为主峰芦芽山 $2787 \mathrm{~m}$, 最低海拔 $1346 \mathrm{~m}$, 年平均 气温4-7 ${ }^{\circ} \mathrm{C}$, 年降水量500-600 mm, 无霜期90-120 天。该区土壤分布复杂, 区域东部是黄土母质发育 而成的灰褐土, 西部以褐土为主。随海拔高度的升 高, 土壤呈现垂直地带性, 依次为山地褐土、山地淋 溶褐土、棕色森林土和亚高山草甸土。芦芽山植被 具有明显的垂直地带性, 从山麓到山顶依次为森林 草原带、落叶阔叶林带、针阔叶混交林带、寒温性 针叶林带、亚高山灌从草甸带。芦芽山东麓西麓基 带不同, 东麓基带为森林草原带, 西麓基带为落叶 阔叶林带。沿海拔梯度, 保护区内优势的灌从群落 主要有沙棘(Hippophae rhamnoides)灌从、山刺玫 (Rosa davurica)灌从、绣线菊(Spiraea spp.)灌丛、虎 榛子 (Ostryopsis davidiana) 灌丛、蒙古荚蒾 (Viburnum mongolicum) 灌从、金花忍冬(Lonicera chrysantha)灌从、银露梅(Potentilla glabra)灌丛和鬼 箭锦鸡儿(Caragana jubata)灌丛(张金屯, 1989)。

\section{2 样品采集方法}

所有样品均采自于2014年7月, 选取芦芽山自 然保护区内14种常见灌木作为研究对象, 即沙棘、 三裂绣线菊(Spiraea trilobata)、土庄绣线菊(S. pubescens)、黄刺玫(Rosa xanthina)、虎榛子、榛(Corylus 
heterophylla)、金花忍冬、蒙古荚蒾、唐古特忍冬 (Lonicera tangutica)、复盆子(Rubus idaeus)、银露梅、 山刺玫、刺果茶蔍子(Ribes burejense)和鬼箭锦鸡儿, 14种灌木取样点的群落类型都为灌丛群落。14种灌 木采样调查基本信息见表1。MacDicken (1997) ${ }^{\circledR}$ 认 为对于区域性生物量表的建立需要30-100株样木, 如果每个径级的样木均匀分布的话, 30株样木是最 合适的。因此我们在每种灌木分布较为集中的区域 从小到大调查30株样木。其中鬼箭锦鸡儿为贴地生 长, 银露梅和三裂绣线菊地上分枝数极多, 难以调 查其基径, 只调查其冠幅(取最大直径和最小直径 的平均值)和株高, 其余11种灌木调查株高、冠幅以 及每株灌木地上所有分枝的基径用以求得每株的总 基径。测量完毕后将植株整株挖出, 深度为根系分 布所达范围。将植株分根、茎、叶三部分储存, 所 有样品带回实验室在 $65{ }^{\circ} \mathrm{C}$ 烘箱内烘干至恒质量, 测得相应的干质量。模型参数的实测区间和相应的 生物量见附录I。

\section{3 数据处理分析}

用SPSS 20.0软件进行生物量模型的拟合。选取 总基径 $\left(D_{\mathrm{t}}\right)$ 、株高 $(H)$ 、冠幅 $(C)$ 、冠幅面积 $(A=$ $\left.\pi(C / 2)^{2}\right)$ 、冠幅体积 $(V=A \times H)$ 、总基径平方与株高 乘积 $\left(D_{\mathrm{t}}^{2} H\right)$ 为变量, 对各器官、地上和总生物量进行 模型拟合。模型采用以下3种类型:

$$
W=a+b X
$$

$$
\begin{aligned}
& W=a+b \ln X \\
& W=a X^{b}
\end{aligned}
$$

其中 $W$ 表示预测变量(本文中指各类生物量), $X$ 表示 拟合变量(如株高、冠幅等), $a 、 b$ 表示拟合参数。根 据决定系数 $\left(R^{2}\right)$ 、估计值的标准误差 (SEE) 以及平均 预估误差 $(M P E)$ 的大小及回归检验显著水平 $(p$ 值)来 评价模型的优劣(曾伟生和唐守正, 2011)。从中选出 拟合度最好、相关最密切的数学模型作为生物量的

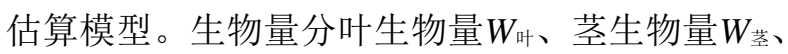

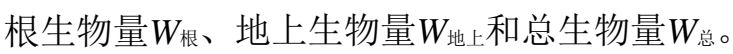

生物量分配测定指标包括: 根冠比(地下生物 量/地上生物量)、叶质比(叶生物量/总生物量)、茎 质比(茎生物量/总生物量)和根质比(根生物量/总生 物量)。分别根据灌木是否带刺对14种灌木进行分 类, 将物种作为随机变量, 对不同种类灌木的根冠 比、叶质比、茎质比和根质比采用单因素方差分析 比较其差异, 所有的数据处理和分析在Excel 2010 和SPSS 20.0中完成。

\section{2 结果}

\section{1 生物量模型的拟合}

经回归分析, 建立了 14 种灌木 3 个器官、地上及 总生物量的最优生物量拟合模型(表2)。除复盆子的 叶生物量模型外, 14 种灌木的最优生物量模型均为 幂函数或线性函数。冠幅面积 $(A)$ 、冠幅体积 $(V)$ 、

\begin{tabular}{|c|c|c|c|c|c|c|}
\hline 物种 & 经度 & 纬度 & 海拔 & 科 & 属 & 是否带刺 \\
\hline Species & Longitude (E) & Latitude $(\mathrm{N})$ & Elevation (m) & Family & Genus & Thorn \\
\hline 沙欶 Hippophae rhamnoides & $112.01^{\circ}$ & $38.68^{\circ}$ & 1499.8 & 胡颓子科 Elaeagnaceae & 沙棘属 Hippophae & 是 Yes \\
\hline 三裂绣线菊 Spiraea trilobata & $111.99^{\circ}$ & $38.69^{\circ}$ & 1535.6 & 蓄薇科 Rosaceae & 绣线菊属 Spiraea & 否 No \\
\hline 黄刺玫 Rosa xanthina & $111.99^{\circ}$ & $38.69^{\circ}$ & 1535.6 & 蓄薇科 Rosaceae & 萻薇属 Rosa & 是 Yes \\
\hline 虎榛子 Ostryopsis davidiana & $111.99^{\circ}$ & $38.69^{\circ}$ & 1535.6 & 桦木科 Betulaceae & 虎榛子属 Ostryopsis & 否 No \\
\hline 榛 Corylus heterophylla & $111.99^{\circ}$ & $38.69^{\circ}$ & 1535.6 & 桦木科 Betulaceae & 榛属 Corylus & 否 No \\
\hline 土庄绣线菊 Spiraea pubescens & $111.98^{\circ}$ & $38.72^{\circ}$ & 1659.9 & 蓄薇科 Rosaceae & 绣线菊属 Spiraea & 否 No \\
\hline 金花忍冬 Lonicera chrysantha & $111.92^{\circ}$ & $38.65^{\circ}$ & 1778.4 & 忍冬科 Caprifoliaceae & 忍冬属 Lonicera & 否 No \\
\hline 蒙古荚蒾 Viburnum mongolicum & $111.92^{\circ}$ & $38.65^{\circ}$ & 1780.0 & 忍冬科 Caprifoliaceae & 荚迷属 Viburnum & 否 No \\
\hline 唐古特忍冬 Lonicera tangutica & $111.92^{\circ}$ & $38.65^{\circ}$ & 1780.0 & 忍冬科 Caprifoliaceae & 忍冬属 Lonicera & 否 No \\
\hline 复盆子 Rubus idaeus & $111.92^{\circ}$ & $38.65^{\circ}$ & 1780.0 & 菩薇科 Rosaceae & 悬钩子属 Rubus & 是 Yes \\
\hline 银露梅 Potentilla glabra & $111.96^{\circ}$ & $38.72^{\circ}$ & 1812.2 & 蓄薇科 Rosaceae & 委陵菜属 Potentilla & 否 No \\
\hline 山刺玫 Rosa davurica & $111.95^{\circ}$ & $38.71^{\circ}$ & 1874.3 & 葍薇科 Rosaceae & 蓄薇属 Rosa & 是 Yes \\
\hline 刺果茶蔍子 Ribes burejense & $111.95^{\circ}$ & $38.71^{\circ}$ & 1891.1 & 虎耳草科 Saxifragaceae & 茶蔍子属 Ribes & 是 Yes \\
\hline 鬼箭锦鸡儿 Caragana jubata & $111.86^{\circ}$ & $38.73^{\circ}$ & 2602.2 & 豆科 Leguminosae & 锦鸡儿属 Caragana & 是 Yes \\
\hline
\end{tabular}

表1 芦芽山14种灌木调查基本信息表

Table 1 The basic information of 14 shrub species in Mountain Luya

(1) MacDicken KG (1997). A guide to monitoring carbon storage in forestry and agroforestry projects. Forest Carbon Monitoring Program, Winrock International Institute for Agricultural Development. 
表2 14种灌木总生物量 $\left(W_{\text {Total }}\right)$ 、地上生物量 $\left(W_{\text {Aboveground }}\right)$ 、根生物量 $\left(W_{\text {Root }}\right)$ 、茎生物量 $\left(W_{\text {Stem }}\right) 、$ 叶生物量 $\left(W_{\text {Leaf }}\right)$ 的最佳拟合模型 $(n=30)$

Table 2 The best estimation models for total biomass ( $\left.W_{\text {Total }}\right)$, aboveground biomass ( $\left.W_{\text {Aboveground }}\right)$, root biomass $\left(W_{\text {Root }}\right)$, stem biomass ( $\left.W_{\text {Stem }}\right)$, and leaf biomass $\left(W_{\text {Leaf }}\right)$ of 14 shrub species $(n=30)$

\begin{tabular}{|c|c|c|c|c|c|c|c|c|}
\hline $\begin{array}{l}\text { 物种名 } \\
\text { Species }\end{array}$ & $\begin{array}{l}\text { 最佳方程 } \\
\text { Best model }\end{array}$ & $\begin{array}{l}\text { 最佳变量 } \\
\text { Best variable }\end{array}$ & $\begin{array}{c}\text { 参数 } a \\
\text { Parameter } \\
\text { variable } a\end{array}$ & $\begin{array}{c}\text { 参数 } b \\
\text { Parameter } \\
\text { variable } b\end{array}$ & $\begin{array}{l}\text { 平均预估误差 } \\
\text { Mean prediction } \\
\text { error }(M P E)(\%)\end{array}$ & $\begin{array}{c}\text { 估计值的标准误差 } \\
\text { Standard error of } \\
\text { estimate (SEE) }\end{array}$ & $R^{2}$ & $p$ \\
\hline \multirow{5}{*}{$\begin{array}{l}\text { 沙棘 } \\
\text { Hippophae rhamnoides }\end{array}$} & $W_{\text {Total }}=a D_{\mathrm{t}}^{2} H+b$ & $D_{\mathrm{t}}^{2} H$ & 0.0020 & 52.34 & 15.48 & 94.78 & 0.91 & $<0.001$ \\
\hline & $W_{\text {Aboveground }}=a D_{\mathrm{t}}^{2} H+b$ & $D_{\mathrm{t}}^{2} H$ & 0.0020 & 40.77 & 17.42 & 85.18 & 0.90 & $<0.001$ \\
\hline & $W_{\text {Root }}=a D_{\mathrm{t}}^{2} H+b$ & $D_{\mathrm{t}}^{2} H$ & 0.0004 & 11.58 & 12.24 & 15.12 & 0.93 & $<0.001$ \\
\hline & $W_{\text {Stem }}=a D_{\mathrm{t}}^{2} H+b$ & $D_{\mathrm{t}}^{2} H$ & 0.0010 & 30.06 & 18.10 & 69.05 & 0.89 & $<0.001$ \\
\hline & $W_{\text {Leaf }}=a D_{\mathrm{t}}^{2} H+b$ & $D_{\mathrm{t}}^{2} H$ & 0.0004 & 10.71 & 17.27 & 18.55 & 0.88 & $<0.001$ \\
\hline \multirow{5}{*}{$\begin{array}{l}\text { 三裂绣线菊 } \\
\text { Spiraea trilobata }\end{array}$} & $W_{\text {Total }}=a V^{b}$ & $V$ & 0.0490 & 0.66 & 0.40 & 0.83 & 0.45 & $<0.001$ \\
\hline & $W_{\text {Aboveground }}=a V^{b}$ & $V$ & 0.0220 & 0.69 & 0.64 & 0.85 & 0.46 & $<0.001$ \\
\hline & $W_{\text {Root }}=a V^{b}$ & $V$ & 0.0300 & 0.60 & 1.17 & 0.86 & 0.39 & $<0.001$ \\
\hline & $W_{\text {Stem }}=a V^{b}$ & $V$ & 0.0120 & 0.72 & 0.95 & 0.96 & 0.42 & $<0.001$ \\
\hline & $W_{\text {Leaf }}=a V^{b}$ & $V$ & 0.0050 & 0.70 & 2.57 & 0.85 & 0.46 & $<0.001$ \\
\hline \multirow{5}{*}{$\begin{array}{l}\text { 黄刺玫 } \\
\text { Rosa xanthina }\end{array}$} & $W_{\text {Total }}=a V^{b}$ & $V$ & 0.0050 & 0.88 & 0.07 & 0.34 & 0.90 & $<0.001$ \\
\hline & $W_{\text {Aboveground }}=a V^{b}$ & $V$ & 0.0030 & 0.88 & 0.11 & 0.32 & 0.93 & $<0.001$ \\
\hline & $W_{\text {Root }}=a V^{b}$ & $V$ & 0.0050 & 0.77 & 0.29 & 0.50 & 0.80 & $<0.001$ \\
\hline & $W_{\text {Stem }}=a V^{b}$ & $V$ & 0.0020 & 0.89 & 0.16 & 0.35 & 0.92 & $<0.001$ \\
\hline & $W_{\text {Leaf }}=a V^{b}$ & $V$ & 0.0010 & 0.86 & 0.40 & 0.33 & 0.92 & $<0.001$ \\
\hline \multirow{5}{*}{$\begin{array}{l}\text { 虎榛子 } \\
\text { Ostryopsis davidiana }\end{array}$} & $W_{\text {Total }}=a V+b$ & $V$ & 0.0010 & 9.28 & 13.66 & 29.28 & 0.90 & $<0.001$ \\
\hline & $W_{\text {Aboveground }}=a V+b$ & $V$ & 0.0008 & 6.25 & 12.87 & 19.43 & 0.91 & $<0.001$ \\
\hline & $W_{\text {Root }}=a V+b$ & $V$ & 0.0002 & 3.03 & 16.66 & 10.56 & 0.86 & $<0.001$ \\
\hline & $W_{\text {Stem }}=a V+b$ & $V$ & 0.0006 & 2.93 & 16.70 & 17.86 & 0.87 & $<0.001$ \\
\hline & $W_{\text {Leaf }}=a V+b$ & $V$ & 0.0002 & 3.32 & 9.14 & 4.03 & 0.94 & $<0.001$ \\
\hline \multirow{5}{*}{$\begin{array}{l}\text { 榛 } \\
\text { Corylus heterophylla }\end{array}$} & $W_{\text {Total }}=a\left(D_{\mathrm{t}}^{2} H\right)^{b}$ & $D_{\mathrm{t}}^{2} H$ & 0.1450 & 0.64 & 0.10 & 0.32 & 0.86 & $<0.001$ \\
\hline & $W_{\text {Aboveground }}=a V^{b}$ & $V$ & 0.0001 & 1.15 & 0.17 & 0.34 & 0.85 & $<0.001$ \\
\hline & $W_{\text {Root }}=a\left(D_{\mathrm{t}}^{2} H\right)^{b}$ & $D_{\mathrm{t}}^{2} H$ & 0.0320 & 0.68 & 0.30 & 0.34 & 0.86 & $<0.001$ \\
\hline & $W_{\text {Stem }}=a\left(D_{\mathrm{t}}^{2} H\right)^{b}$ & $D_{\mathrm{t}}^{2} H$ & 0.0310 & 0.71 & 0.28 & 0.40 & 0.82 & $<0.001$ \\
\hline & $W_{\text {Leaf }}=a V^{b}$ & $V$ & 0.0009 & 0.90 & 0.48 & 0.30 & 0.81 & $<0.001$ \\
\hline \multirow{5}{*}{$\begin{array}{l}\text { 土庄绣线菊 } \\
\text { Spiraea pubescens }\end{array}$} & $W_{\text {Total }}=a V+b$ & $V$ & 0.0010 & 18.90 & 13.81 & 38.03 & 0.89 & $<0.001$ \\
\hline & $W_{\text {Aboveground }}=a V+b$ & $V$ & 0.0020 & 8.02 & 12.84 & 22.11 & 0.91 & $<0.001$ \\
\hline & $W_{\text {Root }}=a V^{b}$ & $V$ & 0.0200 & 0.74 & 0.44 & 0.45 & 0.78 & $<0.001$ \\
\hline & $W_{\text {Stem }}=a V+b$ & $V$ & 0.0020 & 6.18 & 12.95 & 20.92 & 0.92 & $<0.001$ \\
\hline & $W_{\text {Leaf }}=a\left(D_{\mathrm{t}}^{2} H\right)^{b}$ & $D_{\mathrm{t}}^{2} H$ & 0.0110 & 0.53 & 4.33 & 0.46 & 0.71 & $<0.001$ \\
\hline \multirow{5}{*}{$\begin{array}{l}\text { 金花忍冬 } \\
\text { Lonicera chrysantha }\end{array}$} & $W_{\text {Total }}=a\left(D_{\mathrm{t}}^{2} H\right)^{b}$ & $D_{\mathrm{t}}^{2} H$ & 0.3650 & 0.56 & 0.08 & 0.42 & 0.82 & $<0.001$ \\
\hline & $W_{\text {Aboveground }}=a\left(D_{\mathrm{t}}^{2} H\right)^{b}$ & $D_{\mathrm{t}}^{2} H$ & 0.2330 & 0.53 & 0.16 & 0.40 & 0.83 & $<0.001$ \\
\hline & $W_{\text {Root }}=a D_{\mathrm{t}}^{2} H+b$ & $D_{\mathrm{t}}^{2} H$ & 0.0005 & 43.22 & 25.05 & 67.14 & 0.76 & $<0.001$ \\
\hline & $W_{\text {Stem }}=a\left(D_{\mathrm{t}}^{2} H\right)^{b}$ & $D_{\mathrm{t}}^{2} H$ & 0.1360 & 0.56 & 0.20 & 0.41 & 0.83 & $<0.001$ \\
\hline & $W_{\text {Leaf }}=a D_{\mathrm{t}}^{2} H+b$ & $D_{\mathrm{t}}^{2} H$ & 0.0001 & 9.08 & 18.23 & 8.19 & 0.78 & $<0.001$ \\
\hline \multirow{5}{*}{$\begin{array}{l}\text { 蒙古荚蒾 } \\
\text { Viburnum mongolicum }\end{array}$} & $W_{\text {Total }}=a V^{b}$ & $V$ & 0.0060 & 0.88 & 0.12 & 0.32 & 0.92 & $<0.001$ \\
\hline & $W_{\text {Aboveground }}=a V^{b}$ & $V$ & 0.0030 & 0.90 & 0.21 & 0.34 & 0.92 & $<0.001$ \\
\hline & $W_{\text {Root }}=a V^{b}$ & $V$ & 0.0020 & 0.84 & 0.70 & 0.66 & 0.72 & $<0.001$ \\
\hline & $W_{\text {Stem }}=a V^{b}$ & $V$ & 0.0010 & 0.91 & 0.34 & 0.47 & 0.91 & $<0.001$ \\
\hline & $W_{\text {Leaf }}=a V^{b}$ & $V$ & 0.0003 & 0.89 & 1.94 & 0.50 & 0.85 & $<0.001$ \\
\hline \multirow{3}{*}{$\begin{array}{l}\text { 唐古特忍冬 } \\
\text { Lonicera tangutica }\end{array}$} & $W_{\text {Total }}=a\left(D_{\mathrm{t}}^{2} H\right)^{b}$ & $D_{\mathrm{t}}^{2} H$ & 0.2000 & 0.54 & 0.30 & 0.34 & 0.80 & $<0.001$ \\
\hline & $W_{\text {Aboveground }}{ }^{f}=a V^{b}$ & $V$ & 0.0070 & 0.80 & 0.49 & 0.33 & 0.84 & $<0.001$ \\
\hline & $W_{\text {Root }}=a D_{\mathrm{t}}^{2} H+b$ & $D_{\mathrm{t}}^{2} H$ & 0.0004 & 6.22 & 11.48 & 5.13 & 0.73 & $<0.001$ \\
\hline
\end{tabular}


表2 (续) Table 2 (continued)

\begin{tabular}{|c|c|c|c|c|c|c|c|c|}
\hline $\begin{array}{l}\text { 物种名 } \\
\text { Species }\end{array}$ & $\begin{array}{l}\text { 最佳方程 } \\
\text { Best model }\end{array}$ & $\begin{array}{l}\text { 最佳变量 } \\
\text { Best variable }\end{array}$ & $\begin{array}{c}\text { 参数 } a \\
\text { Parameter } \\
\text { variable } a \\
\end{array}$ & $\begin{array}{c}\text { 参数 } b \\
\text { Parameter } \\
\text { variable } b \\
\end{array}$ & $\begin{array}{l}\text { 平均预估误差 } \\
\text { Mean prediction } \\
\text { error }(M P E)(\%)\end{array}$ & $\begin{array}{c}\text { 估计值的标准误差 } \\
\text { Standard error of } \\
\text { estimate (SEE) }\end{array}$ & $R^{2}$ & $p$ \\
\hline \multirow{2}{*}{$\begin{array}{l}\text { 唐古特忍冬 } \\
\text { Lonicera tangutica }\end{array}$} & $W_{\text {Stem }}=a\left(D_{\mathrm{t}}^{2} H\right)^{b}$ & $\overline{D_{\mathrm{t}}^{2} H}$ & 0.0630 & 0.59 & 0.74 & 0.41 & 0.77 & $<0.001$ \\
\hline & $W_{\text {Leaf }}=a V^{b}$ & $V$ & 0.0050 & 0.68 & 2.30 & 0.29 & 0.81 & $<0.001$ \\
\hline \multirow{5}{*}{$\begin{array}{l}\text { 复盆子 } \\
\text { Rubus idaeus }\end{array}$} & $W_{\text {Total }}=a V+b$ & $V$ & 0.0006 & 12.52 & 10.18 & 6.76 & 0.84 & $<0.001$ \\
\hline & $W_{\text {Aboveground }}=a V+b$ & V & 0.0004 & 10.71 & 10.63 & 5.59 & 0.80 & $<0.001$ \\
\hline & $W_{\text {Root }}=a V+b$ & $V$ & 0.0000 & 1.81 & 13.92 & 1.92 & 0.83 & $<0.001$ \\
\hline & $W_{\text {Stem }}=a V+b$ & $V$ & 0.0002 & 4.59 & 14.17 & 3.43 & 0.73 & $<0.001$ \\
\hline & $W_{\text {Leaf }}=a \ln V+b$ & V & 14.1660 & -69.23 & 11.41 & 3.24 & 0.77 & $<0.001$ \\
\hline \multirow{5}{*}{$\begin{array}{l}\text { 银露梅 } \\
\text { Potentilla glabra }\end{array}$} & $W_{\text {Total }}=a A+b$ & $A$ & 0.1310 & -6.74 & 19.50 & 42.11 & 0.82 & $<0.001$ \\
\hline & $W_{\text {Aboveground }}=a A+b$ & $A$ & 0.0770 & -2.81 & 19.75 & 25.62 & 0.81 & $<0.001$ \\
\hline & $W_{\text {Root }}=a A+b$ & A & 0.0540 & -3.93 & 22.54 & 19.43 & 0.78 & $<0.001$ \\
\hline & $W_{\text {Stem }}=a A+b$ & $A$ & 0.0680 & -3.26 & 20.73 & 23.16 & 0.80 & $<0.001$ \\
\hline & $W_{\text {Leaf }}=a A+b$ & $A$ & 0.0100 & 0.44 & 20.89 & 3.76 & 0.74 & $<0.001$ \\
\hline \multirow{5}{*}{$\begin{array}{l}\text { 山刺玫 } \\
\text { Rosa davurica }\end{array}$} & $W_{\text {Total }}=a\left(D_{\mathrm{t}}^{2} H\right)^{b}$ & $D_{\mathrm{t}}^{2} H$ & 0.0610 & 0.69 & 0.11 & 0.29 & 0.88 & $<0.001$ \\
\hline & $W_{\text {Aboveground }}=a\left(D_{\mathrm{t}}^{2} H\right)^{b}$ & $D_{\mathrm{t}}^{2} H$ & 0.0420 & 0.67 & 0.22 & 0.35 & 0.83 & $<0.001$ \\
\hline & $W_{\text {Root }}=a\left(D_{\mathrm{t}}^{2} H\right)^{b}$ & $D_{\mathrm{t}}^{2} H$ & 0.0230 & 0.70 & 0.37 & 0.40 & 0.80 & $<0.001$ \\
\hline & $W_{\text {Stem }}=a\left(D_{\mathrm{t}}^{2} H\right)^{b}$ & $D_{\mathrm{t}}^{2} H$ & 0.0250 & 0.70 & 0.27 & 0.36 & 0.84 & $<0.001$ \\
\hline & $W_{\text {Leaf }}=a A+b$ & $A$ & 0.0200 & -0.87 & 7.35 & 1.94 & 0.95 & $<0.001$ \\
\hline \multirow{5}{*}{$\begin{array}{l}\text { 刺果茶蔍子 } \\
\text { Ribes burejense }\end{array}$} & $W_{\text {Total }}=a V^{b}$ & V & 0.5500 & 0.41 & 0.36 & 0.43 & 0.64 & $<0.001$ \\
\hline & $W_{\text {Aboveground }}=a V+b$ & V & 0.0004 & 12.39 & 16.40 & 12.07 & 0.66 & $<0.001$ \\
\hline & $W_{\text {Root }}=a A^{b}$ & $A$ & 0.9380 & 0.46 & 1.39 & 0.64 & 0.47 & $<0.001$ \\
\hline & $W_{\text {Stem }}=a V+b$ & V & 0.0002 & 9.22 & 15.79 & 9.06 & 0.70 & $<0.001$ \\
\hline & $W_{\text {Leaf }}=a V^{b}$ & $V$ & 0.0160 & 0.55 & 3.89 & 0.63 & 0.60 & $<0.001$ \\
\hline \multirow{5}{*}{$\begin{array}{l}\text { 鬼箭锦鸡儿 } \\
\text { Caragana jubata }\end{array}$} & $W_{\text {Total }}=a A+b$ & $A$ & 0.2680 & -10.27 & 12.92 & 48.69 & 0.93 & $<0.001$ \\
\hline & $W_{\text {Aboveground }}=a A+b$ & $A$ & 0.1590 & -3.65 & 16.51 & 38.04 & 0.88 & $<0.001$ \\
\hline & $W_{\text {Root }}=a A+b$ & $A$ & 0.1100 & -6.63 & 11.13 & 16.30 & 0.95 & $<0.001$ \\
\hline & $W_{\text {Stem }}=a A+b$ & $A$ & 0.1200 & -2.83 & 16.74 & 29.02 & 0.88 & $<0.001$ \\
\hline & $W_{\text {Leaf }}=a A+b$ & $A$ & 0.0400 & -0.82 & 21.53 & 12.27 & 0.81 & $<0.001$ \\
\hline
\end{tabular}

$A$, 冠幅面积, $A=\pi(C / 2)^{2}\left(\mathrm{~cm}^{2}\right) ; V$, 冠幅体积, $V=A \times H\left(\mathrm{~cm}^{3}\right) ; D_{\mathrm{t}}^{2} H$, 总基径平方与树高乘积, $\left.D_{\mathrm{t}}^{2} H=D_{\mathrm{t}} \times D_{\mathrm{t}} \times H\left((\mathrm{~mm})^{2}\right) \times \mathrm{cm}\right)$ 。 $a$ 和 $b$ 是参数。

$A$, canopy area, $A=\pi(C / 2)^{2}\left(\mathrm{~cm}^{2}\right) ; V$, canopy volume, $V=A \times H\left(\mathrm{~cm}^{3}\right) ; D_{\mathrm{t}}^{2} H$, the sum of the square of total base diameter multiply stem heigh, $D_{\mathrm{t}}^{2} H=D_{\mathrm{t}} \times D_{\mathrm{t}} \times$ $H\left(\left(\mathrm{~mm}^{2}\right) \times \mathrm{cm}\right) . a$ and $b$ are parameter variables.

总基径平方与树高乘积 $\left(D_{\mathrm{t}}^{2} H\right)$ 是生物量方程的最佳 预测变量。所有灌木生物量的拟合方程都表现出较 好的拟合效果 $(p<0.001 ; M P E<25.1 \%)$, 并且除三 裂绣线菊和刺果茶蔍子外, 其余12种灌木的生物量 拟合方程 $R^{2}$ 都在 0.7 以上。从模型的篮选结果来看, 沙棘、金花忍冬的所有生物量方程以及山刺玫、榛、 唐古特忍冬的大部分生物量方程其预测变量都是 $D_{\mathrm{t}}^{2} \mathrm{H}$, 银露梅、鬼箭锦鸡儿所有生物量方程预测变 量都为 $A$, 其他种生物量方程最佳预测变量大多为 $V$ 。图1以不同种的总生物量为例, 对 3 种预测变量、 两种方程形式构成的6种主要生物量方程进行展示。

\section{2 生物量分配}

表3统计了芦芽山14种灌木的根冠比、叶质比、 茎质比和根质比的基本特征(范围、均值和中位数)。
整体而言, 芦芽山14种常见灌木的平均根冠比为 0.61 (0.31-1.05), 平均叶质比为 0.17 (0.05-0.44), 平 均茎质比为 $0.48(0.36-0.57)$, 平均根质比为 0.35 (0.20-0.48)。其中, 对根冠比而言, 除金花忍冬 (1.05)外, 其他 13 种灌木的平均根冠比都小于 1 。此 外, 平均叶质比表现出最大的种间差异, 叶质比最 大的复盆子 $(0.44)$ 高出叶质比最小的土庄绣线菊 (0.05) 将近8倍; 平均茎质比的种间差异最小, 而平 均根质比的种间差异居中。

依照灌木是否带刺, 我们将14种灌木分为带刺 (6种)与不带刺(8种)灌木两类。单因素方差分析表 明, 不带刺灌木的根冠比、茎质比和根质比显著大 于带刺灌木的根冠比、茎质比和根质比, 而其叶质 比则显著小于带刺灌木的叶质比(图2)。 

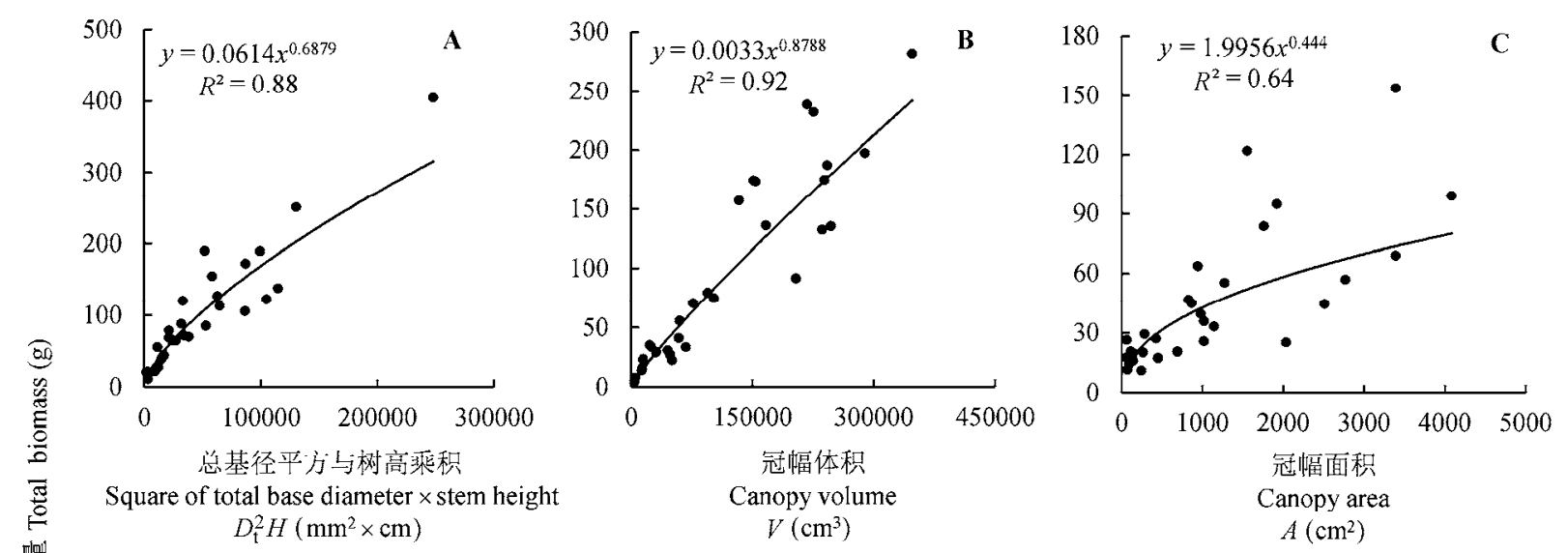

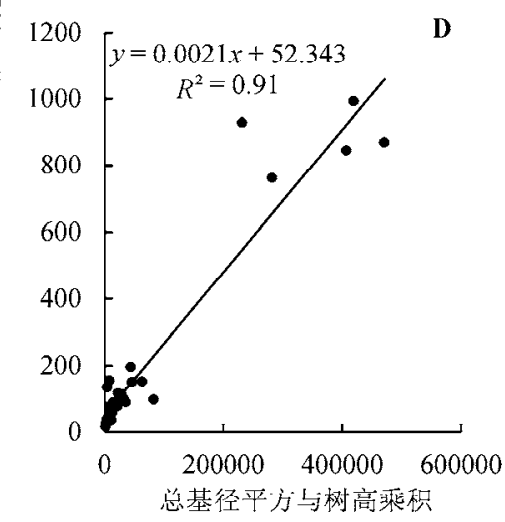

Square of total base diameter $\times$ stem height $D_{\mathrm{t}}^{2} H\left(\mathrm{~mm}^{2} \times \mathrm{cm}\right)$
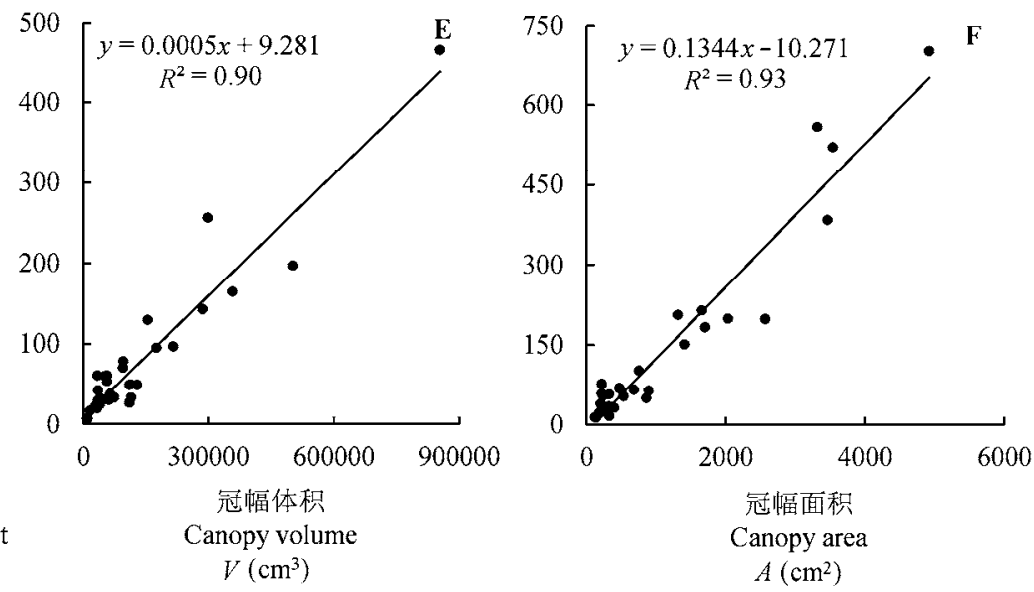

图1 芦芽山6种灌木总生物量最佳拟合模型。A, 刺玫。 $\mathbf{B}$, 蒙古荚蒾。C, 刺果茶蔍子。 D, 沙棘。 E, 虎榛子。F, 鬼箭锦鸡儿。 Fig. 1 The best biomass estimation model of six shrub species in Mountain Luya. A, Rosa davurica. B, Viburnum mongolicum. C, Ribes burejense. D, Hippophae rhamnoides. E, Ostryopsis davidiana. F, Caragana jubata.

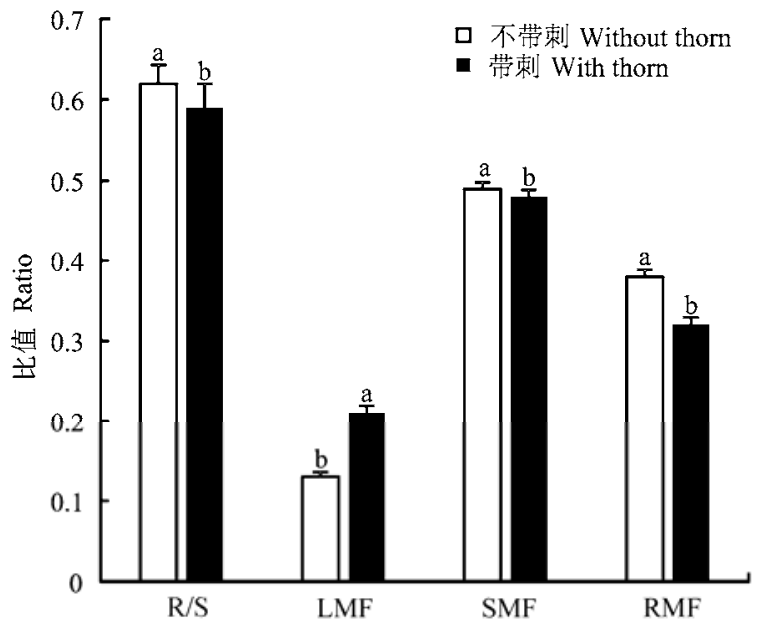

图2 带刺与不带刺灌木之间叶质比(LMF)、茎质比(SMF)、 根质比(RMF)、根冠比(R/S)的比较(平均值土标准误差)。用单 因素方差分析(Tukey post hoc)检验不同类别之间的差异; 显 著性差异 $(p<0.05)$ 用不同字母标记。

Fig. 2 Comparisons of leaf biomass fraction (LMF), stem biomass fraction (SMF), root biomass fraction (RMF), root/shoot ratio $(\mathrm{R} / \mathrm{S})$ between shrubs with and without thorn (mean $\pm S E$ ). Differences between each group were tested using a one-way ANOVA with a Tukey post hoc test of significance; significance different at $p<0.05$ was indicated by different letters.

\section{3 讨论}

\section{1 生物量模型}

曾珍英等(2005)在对灌木生物量的研究中发现, 用单个变量来模拟生物量回归方程是不理想的，只 有各个变量的适当组合才能比较准确地估算灌木生 物量。卢振龙和龚孝生(2009)研究认为不同形态指 标的组合代表了一定的物理意义，例如树高 $(H)$ 和 树干直径 $(D)$ 的乘积 $(H \times D)$ 可理解为树干纵断面周 长, $\mathrm{CH}$ 可以理解为投影纵断面积，而树干直径平方 和树高的乘积 $\left(D^{2} H\right)$ 可近似地理解为树干纵断面积。 本研究表明, 不同灌木种的最优生物量估算模型不 同，而相同物种的各个生物量估测模型不论是方程 的形式还是预测变量几乎都是一致的, 这与种与种 之间的差异有关。 $A 、 V 、 D_{\mathrm{t}}^{2} H$ 是拟合生物量方程的 3 个最佳测量指标。其中, 鬼箭锦鸡儿和银露梅的预 测变量为 $A$, 其外部形态低矮(两种灌木平均高度 40 $\mathrm{cm}$ )，且分枝较多(两种灌木平均分葟数4.8); 而沙 
表3 14种灌木的根冠比、叶质比、茎质比及根质比的统计特征

Table 3 The descriptive statistics of root/shoot ratio (R/S), leaf biomass fraction (LMF), stem biomass fraction (SMF), and root biomass fraction (RMF) of 14 shrub species

\begin{tabular}{|c|c|c|c|c|c|c|c|c|c|c|c|c|}
\hline \multirow{2}{*}{$\begin{array}{l}\text { 物种 } \\
\text { Species }\end{array}$} & \multicolumn{3}{|c|}{ 根冠比 R/S } & \multicolumn{3}{|c|}{ 叶质比 LMF } & \multicolumn{3}{|c|}{ 茎质比 SMF } & \multicolumn{3}{|c|}{ 根质比 RMF } \\
\hline & $\begin{array}{l}\text { 范围 } \\
\text { Range }\end{array}$ & $\begin{array}{c}\text { 平均值 } \pm \\
\text { 标准偏差 } \\
\text { Mean } \pm S D\end{array}$ & $\begin{array}{l}\text { 中位值 } \\
\text { Median }\end{array}$ & $\begin{array}{l}\text { 范围 } \\
\text { Range }\end{array}$ & $\begin{array}{c}\text { 平均值 } \pm \\
\text { 标准偏差 } \\
\text { Mean } \pm S D\end{array}$ & $\begin{array}{l}\text { 中位值 } \\
\text { Median }\end{array}$ & $\begin{array}{l}\text { 范围 } \\
\text { Range }\end{array}$ & $\begin{array}{c}\text { 平均值 } \pm \\
\text { 标准偏差 } \\
\text { Mean } \pm S D\end{array}$ & $\begin{array}{l}\text { 中位值 } \\
\text { Median }\end{array}$ & $\begin{array}{l}\text { 范围 } \\
\text { Range }\end{array}$ & $\begin{array}{c}\text { 平均值 } \pm \\
\text { 标准偏差 } \\
\text { Mean } \pm S D\end{array}$ & $\begin{array}{l}\text { 中位值 } \\
\text { Median }\end{array}$ \\
\hline $\begin{array}{l}\text { 沙棘 } \\
\text { Hippophae } \\
\text { rhamnoides }\end{array}$ & $0.06-0.59$ & $0.31 \pm 0.14$ & 0.29 & $0.07-0.30$ & $0.20 \pm 0.06$ & 0.20 & $0.39-0.79$ & $0.57 \pm 0.11$ & 0.54 & $0.06-0.37$ & $0.23 \pm 0.08$ & 0.22 \\
\hline $\begin{array}{l}\text { 三裂绣线菊 } \\
\text { Spiraea } \\
\text { trilobata }\end{array}$ & $0.32-1.24$ & $0.57 \pm 0.21$ & 0.51 & $0.04-0.33$ & $0.17 \pm 0.09$ & 0.15 & $0.25-0.67$ & $0.48 \pm 0.12$ & 0.48 & $0.24-0.55$ & $0.35 \pm 0.07$ & 0.34 \\
\hline $\begin{array}{l}\text { 黄刺玫 } \\
\text { Rosa xanthina }\end{array}$ & $0.19-2.00$ & $0.55 \pm 0.34$ & 0.45 & $0.09-0.29$ & $0.19 \pm 0.05$ & 0.18 & $0.24-0.67$ & $0.48 \pm 0.09$ & 0.47 & $0.16-0.67$ & $0.33 \pm 0.11$ & 0.31 \\
\hline $\begin{array}{l}\text { 虎榛子 } \\
\text { Ostryopsis } \\
\text { davidiana }\end{array}$ & $0.16-0.97$ & $0.42 \pm 0.15$ & 0.40 & $0.09-0.45$ & $0.24 \pm 0.08$ & 0.23 & $0.35-0.58$ & $0.47 \pm 0.06$ & 0.48 & $0.14-0.49$ & $0.29 \pm 0.07$ & 0.29 \\
\hline $\begin{array}{l}\text { 榛 } \\
\text { Corylus } \\
\text { heterophylla }\end{array}$ & $0.28-1.24$ & $0.53 \pm 0.22$ & 0.47 & $0.10-0.34$ & $0.24 \pm 0.07$ & 0.23 & $0.26-0.56$ & $0.43 \pm 0.07$ & 0.42 & $0.22-0.55$ & $0.34 \pm 0.08$ & 0.32 \\
\hline $\begin{array}{l}\text { 土庄绣线菊 } \\
\text { Spiraea } \\
\text { pubescens }\end{array}$ & $0.45-1.42$ & $0.72 \pm 0.27$ & 0.63 & $0.01-0.10$ & $0.05 \pm 0.03$ & 0.05 & $0.37-0.67$ & $0.54 \pm 0.08$ & 0.57 & $0.31-0.59$ & $0.41 \pm 0.08$ & 0.39 \\
\hline $\begin{array}{l}\text { 金花忍冬 } \\
\text { Lonicera } \\
\text { chrysantha }\end{array}$ & $0.24-2.42$ & $1.05 \pm 0.54$ & 0.98 & $0.05-0.26$ & $0.10 \pm 0.04$ & 0.10 & $0.20-0.72$ & $0.42 \pm 0.13$ & 0.39 & $0.19-0.71$ & $0.48 \pm 0.13$ & 0.49 \\
\hline $\begin{array}{l}\text { 蒙古英蒾 } \\
\text { Viburnum } \\
\text { mongolicum }\end{array}$ & $0.16-2.06$ & $0.67 \pm 0.45$ & 0.54 & $0.04-0.23$ & $0.10 \pm 0.04$ & 0.10 & $0.28-0.76$ & $0.53 \pm 0.11$ & 0.54 & $0.14-0.67$ & $0.37 \pm 0.13$ & 0.35 \\
\hline $\begin{array}{l}\text { 唐古特忍冬 } \\
\text { Lonicera } \\
\text { tangutica }\end{array}$ & $0.12-1.84$ & $0.67 \pm 0.38$ & 0.54 & $0.06-0.24$ & $0.13 \pm 0.04$ & 0.12 & $0.28-0.65$ & $0.50 \pm 0.10$ & 0.52 & $0.11-0.65$ & $0.37 \pm 0.12$ & 0.35 \\
\hline $\begin{array}{l}\text { 复盆子 } \\
\text { Rubus idaeus }\end{array}$ & $0.11-0.54$ & $0.26 \pm 0.10$ & 0.23 & $0.26-0.67$ & $0.44 \pm 0.10$ & 0.43 & $0.17-0.61$ & $0.36 \pm 0.12$ & 0.35 & $0.10-0.35$ & $0.20 \pm 0.06$ & 0.19 \\
\hline $\begin{array}{l}\text { 银露梅 } \\
\text { Potentilla } \\
\text { glabra }\end{array}$ & $0.29-2.54$ & $0.76 \pm 0.46$ & 0.64 & $0.04-0.21$ & $0.11 \pm 0.05$ & 0.09 & $0.25-0.67$ & $0.49 \pm 0.11$ & 0.51 & $0.22-0.72$ & $0.41 \pm 0.12$ & 0.39 \\
\hline $\begin{array}{l}\text { 山刺玫 } \\
\text { Rosa davuric }\end{array}$ & $0.36-1.29$ & $0.71 \pm 0.24$ & 0.67 & $0.04-0.19$ & $0.11 \pm 0.04$ & 0.10 & $0.37-0.62$ & $0.49 \pm 0.07$ & 0.48 & $0.26-0.56$ & $0.40 \pm 0.08$ & 0.40 \\
\hline $\begin{array}{l}\text { 刺果茶蔍子 } \\
\text { Ribes } \\
\text { burejense }\end{array}$ & $0.27-1.89$ & $0.69 \pm 0.44$ & 0.52 & $0.02-0.35$ & $0.13 \pm 0.07$ & 0.11 & $0.24-0.67$ & $0.49 \pm 0.12$ & 0.52 & $0.21-0.65$ & $0.38 \pm 0.13$ & 0.34 \\
\hline $\begin{array}{l}\text { 鬼箭锦鸡儿 } \\
\text { Caragana } \\
\text { jubata }\end{array}$ & $0.16-1.54$ & $0.60 \pm 0.30$ & 0.58 & $0.08-0.34$ & $0.18 \pm 0.07$ & 0.17 & $0.26-0.68$ & $0.47 \pm 0.09$ & 0.46 & $0.14-0.61$ & $0.36 \pm 0.10$ & 0.37 \\
\hline 整体 Total & & $0.61 \pm 0.38$ & & & $0.17 \pm 0.11$ & & & $0.48 \pm 0.11$ & & & $0.35 \pm 0.12$ & \\
\hline
\end{tabular}

棘、榛、山刺玫、金花忍冬其预测变量为 $D_{\mathrm{t}}^{2} H$, 其 外部形态直立且分枝数较少(平均树高和分苜数分 别为99 $\mathrm{cm}$ 和3.5); 而其他灌木的最佳拟合变量多为 $V$ 。曾慧卿等(2006)研究表明冠幅和高度的乘积能较 为精确地估算聯木(Loropetalum chinense)的地上生 物量, 同时也体现了皪木地上形态近似圆柱形的形 态特征。由实际经验来看, 基径和树高相对于冠幅 来说是两个测量误差较小、拟合方程效果较好的变 量, 因此, 在预测生物量上常用这两个指标作为拟 合变量(林伟等, 2010)。本研究中生物量分析所得模 型均建立在研究区内的植株调查的基础上, 对于灌 木种株高、基径调查范围以外或区域以外的应用需 通过验证。

\section{2 生物量分配}

在植物的整个生长发育过程中, 生长、繁殖和 防御等各种功能对有限的资源始终存在着竞争问 题。植物如何协调各种功能之间资源的分配，即植 物在生长过程中如何将有限的资源分配到根、茎、 叶各器官中反映了植物的生存策略(张大勇, 2010)。 在以往对生物量分配的研究中, 更多地集中在对乔 木物种的研究, 对灌木种的研究较少(Pooter et al., 2012)。本研究中, 芦芽山的14种灌木除金花忍冬根 冠比大于 1 外, 其余灌木种根冠比都小于 1 , 表明芦 芽山灌木更多将光合产物分配到地上部分。这与 Hilbert和Canadell (1995)的研究结果不同, 其发现 地中海地区 10 种常绿灌木的平均根冠比为 2.06 。此 
外, 芦芽山 14 种灌木的平均叶质比为 0.17 , 茎质比 为 0.48 , 都大于Hilbert和Canadell (1995)的中灌木的 平均叶质比(0.07)和平均茎质比(0.31), 表明不同生 活型(落叶与常绿)的灌木种在资源分配上的策略不 同。

刺是植物在与环境的相互作用过程中逐渐进化 形成的一种性状特征, 是植物适应环境的一种外在 表现。植物的刺有防止动物侵害、抵御干旱以及促 进种子传播等功能(贺猛和米楷, 2009)。前人研究多 表明干旱环境条件下的灌木种往往会出现带刺、根 深等特殊的形态特征(易海燕, 2000), 说明带刺灌木 更能在干旱的条件下生存。在我们调查的14种灌木 中, 有6种灌木带刺, 可见刺是芦芽山常见灌木适应 当地环境的一种性状特征。因此, 我们将芦芽山灌 木按照带刺/不带刺分为两类, 发现不带刺灌木和 带刺灌木在生物量分配上(根冠比、叶质比、茎质比 和根质比)存在显著差异, 尤其是叶质比, 带刺灌木 显著小于不带刺灌木, 这可能是带刺灌木对干旱环 境适应的一种表现。这也与Pooter等(2012)的研究表 明随着水分利用率的增加叶质比逐渐降低的结果相 一致。

基金项目中国科学院战略性先导科技专项 (XDA05050302)和国家自然科学基金(31470498)。

致谢 感谢山西芦芽山国家级自然保护区郭建㭉副 局长、宫树龙主任在野外调查中给予的协助。

\section{参考文献}

Bloom AJ, Chapin FS, Mooney HA (1985). Resource limitation in plants: An economic analogy. Annual Review of Ecology and Systematics, 1, 363-392.

Cai Z, Liu QJ, Ouyang QL (2006). Estimation model for biomass of shrubs in Qianyanzhou. Journal of Central South Forestry University, 26(3), 15-23. (in Chinese with English abstract) [蔡哲, 刘琪璟, 欧阳球林 (2006). 千烟洲 实验区几种灌木生物量估算模型的研究. 中南林学院 学报, 26(3), 15-23.]

Cheng DL (2007). Plant Allometric Study of Biomass Allocation Pattern and Biomass Production Rates. PhD dissertation, Lanzhou University, Lanzhou. 40-42. (in Chinese with English abstract). [程栋梁 (2007). 植物生物量分配 模式与生长速率的相关规律研究. 博士学位论文, 兰州 大学, 兰州. 40-42.]

Chen XL, Ma QY, Kang FF, Cao WQ, Zhang GH, Chen ZW (2002). Studies on biomass and productivity of typical shrubs in Taiyue Mountain, Shanxi Province. Forest Re- search, 15, 304-309. (in Chinese with English abstract) [陈遐林, 马钦彦, 康峰峰, 曹文强, 张国华, 陈宗伟 (2002). 山西太岳山典型灌木林生物量及生产力研究. 林业科学研究, 15, 304-309.]

Enquist BJ, Niklas KJ (2002). Global allocation rules for patterns of biomass portioning in seed plants. Science, 295, 1517-1520.

Fang JY, Liu GH, Zhu B, Wang XK, Liu SH (2006). Carbon cycling of three temperate forest ecosystems in Mountain Dongling, Beijing. Scientia Sinica Terrae, 36, 533-543. (in Chinese) [方精云, 刘国华, 朱彪, 王效科, 刘绍辉 (2006). 北京东灵山三种温带森林生态系统的碳循环. 中国科学(地球科学), 36, 533-543.]

He M, Mi K (2009). Study on the common plants with thorns in Shandong Province. Journal of Anhui Agricultural Sciences, 37, 1703 -1705. (in Chinese with English abstract) [贺猛, 米楷 (2009). 山东省常见带刺植物研究. 安徽 农业科学, 37, 1703-1705.]

Hilbert DW, Canadell J (1995). Biomass partitioning and resource allocation of plants from mediterranean-type ecosystems: Possible responses to elevated atmospheric $\mathrm{CO}_{2}$. In: José MM, Walter $\mathrm{CO}$ eds. Global Change and Mediterranean-Type Ecosystems. Springer, Berlin. 76-101.

Jiang FQ, Lu FY (1982). The model of aboveground biomass of Caragana microphylla brush. Acta Ecologica Sinica, 2, 103-110. (in Chinese with English abstract) [姜凤歧, 卢 凤勇 (1982). 小叶锦鸡儿灌丛地上生物量的预测模式. 生态学报, 2, 103-110.]

Lei L (2012). Distribution Character of Alpine Shrubs Biomass Along an Elevation Gradient in Qilian Mountains. Master degree dissertation, Gansu Agriculture University, Lanzhou. 12-21. (in Chinese with English abstract) [雷蕾 (2012). 祁连山高山灌丛生物量沿海拔梯度分配特征研 究. 硕士学位论文, 甘肃农业大学, 兰州. 12-21.]

Li XD, Zhang CP, Fu H (2012). Seasonal dynamics of root-shoot ratio and the effect of factors in grazed and ungrazed grasslands of the Loess Plateau. Acta Prataculture Sinica, 21, 307-312. (in Chinese with English abstract) [李 旭东, 张春平, 傅华 (2012). 黄土高原典型草原地根冠 比的季节动态及其影响因素. 草业学报, 21, 307-312.]

Lin W, Li JS, Zheng BF, Guo JM, Hu LL (2010). Models for estimating biomass of twelve shrub species in Jinggang Mountain Nature Reserve. Journal of Wuhan Botanical Research, 28, 725-729. (in Chinese with English abstract) [林伟, 李俊生, 郑博福, 郭建明, 胡理乐 (2010). 井冈 山自然保护区12种常见灌木生物量的估测模型. 武汉 植物学研究, 28, 725-729.]

Liu CC, Wei YF, Liu YG, Guo K (2009). Biomass of canopy and shrub layers of karst forests in Puding, Guizhou, China. Chinese Journal of Plant Ecology, 33, 698-705. (in 
Chinese with English abstract) [刘长成, 魏雅芬, 刘玉国, 郭柯 (2009). 贵州普定喀斯特次生林乔灌层地上生物 量. 植物生态学报, 33, 698-705.]

Liu XL, Liu SR, Su YM, Cai XH, Ma QY (2006). Aboveground biomass of Quercus aquifolioides shrub community and its responses to altitudinal gradients in Balangshan Mountain, Sichuan Province. Scientia Silvae Sinicae, 42(2), 1-7. (in Chinese with English abstract) [刘兴良, 刘 世荣, 宿以明, 蔡小虎, 马钦彦 (2006). 巴郎山川滇高 山栎灌从地上生物量及其对海拔梯度的响应. 林业科 学, 42(2), 1-7.]

Lu ZL, Gong XS (2009). Progress on the research of shrub biomass estimation. Forest Inventory and Planning, 34(4), 37-45. (in Chinese with English abstract) [卢振龙, 龚孝 生 (2009). 灌木生物量测定的研究进展. 林业调查规 划, 34(4), 37-45.]

Pooter H, Niklas KJ, Reich PB, Oleksyn J, Poot P, Mommer L (2012). Biomass allocation to leaves, stems and roots: Meta-analyses of interspecific variation and environmental control. New Phytologist, 193, 30-50.

Salis SM, Assis MA, Mattos PP, Pião ACS (2006). Estimating the aboveground biomass and wood volume of savanna woodlands in Brazil's Pantanal wetlands based on allometric correlations. Forest Ecology and Management, 228, 61-68.

Whittaker RH (1982). Net production relations of shrubs in the Great Smoky Mountains. Ecology, 43, 357-377.

Yi HY (2010). Study on Soil Microorganism and Soil Enzyme Activity of Different Vegetation in the Mountain ForestsThe Arid Valley Ecotone in the Upper Reach of Minjiang River. Master degree dissertation, Sichuan Agricultural University, Ya'an, Sichuan. 14-17. (in Chinese with English abstract)) [易海燕 (2010). 岷江上游山地森林-干旱河谷 交错带不同植被类型土壤微生物及土壤酶活性的研究. 硕士学位论文, 四川农业大学, 四川雅安. 14-17.]

Yu HS, Li BJ, Zhang BW, Yao HL (2003). The discussion of major ecological function. Inner Mongolia Forestry Science \& Technology, (4), 15-18. (in Chinese) [俞海生, 李 宝军, 张宝文, 姚洪林 (2003). 灌木林主要生态作用的 探讨. 内蒙古林业科技, (4), 15-18.]

Zeng HQ, Liu QJ, Ma ZQ, Zeng ZY (2006). The regression model of Loropetalum chinense biomass based on canopy diameter and plant height. Journal of Nanjing Forestry University (Natural Sciences Edition), 30(4), 101-104. (in Chinese with English abstract) [曾慧卿, 刘琪璟, 马泽清, 曾珍英 (2006). 基于冠幅及植株高度的聯木生物量回 归模型. 南京林业大学学报(自然科学版), 30(4), 101104.]

Zeng HQ, Liu QJ, Feng ZW, Ma ZQ, Hu LL (2007). Estimation models of understory shrub and their applications in red soil hilly region. Chinese Journal of Applied Ecology, 18, 2185-2190. (in Chinese with English abstract) [曾慧卿, 刘琪璟, 冯宗炜, 马泽清, 胡理乐 (2007). 红壤丘陵区 林下灌木生物量估算模型的建立及其应用. 应用生态 学报, 18, 2185-2190.]

Zeng ZY, Liu QJ, Zeng HQ (2005). Study of biomass models of some kinds of shrubs in Qianyanzhou, Jiangxi Province. Journal of Fujian Forestry Science and Technology, 32(4), 68-72. (in Chinese with English abstract) [曾珍英, 刘琪 璟, 曾慧卿 (2005). 江西千烟洲几种灌木生物量模型的 研究. 福建林业科技, 32(4), 68-72.]

Zeng WS, Tang SZ (2011). Goodness evaluation and precision analysis of tree biomass equations. Scientia Silvae Sinicae, 47(11), 106-113. (in Chinese with English abstract) [曾伟 生, 唐守正 (2011). 立木生物量方程的优度评价和精度 分析. 林业科学, 47(11), 106-113.]

Zhang DY (2010). Researches on Theoretical Ecology. Higher Education Press, Beijing. 48. (in Chinese) [张大勇 (2010). 理论生态学研究. 高等教育出版社, 北京. 48.]

Zhang HQ, Liu QJ, Lu PL, Yu Q, Zeng HQ (2005). Biomass estimation of several common shrubs in Qianyanzhou experimental station. Forest Inventory and Planning, 30(5), 43-49. (in Chinese with English abstract) [张海清, 刘琪 璟, 陆佩玲, 于强, 曾慧卿 (2005). 千烟洲试验站几种 常见灌木生物量估测. 林业调查规划, 30(5), 43-49.]

Zhang JT (1989). Vertical zones of vegetation in Luya Mountain in Shanxi Province. Scientia Geographica Since, 9, 346-353. (in Chinese with English abstract) [张金屯 (1989). 山西芦芽山植被垂直带的划分. 地理科学, 9, 346-353.]

责任编委: 谢宗强 责任编辑: 王 葳 


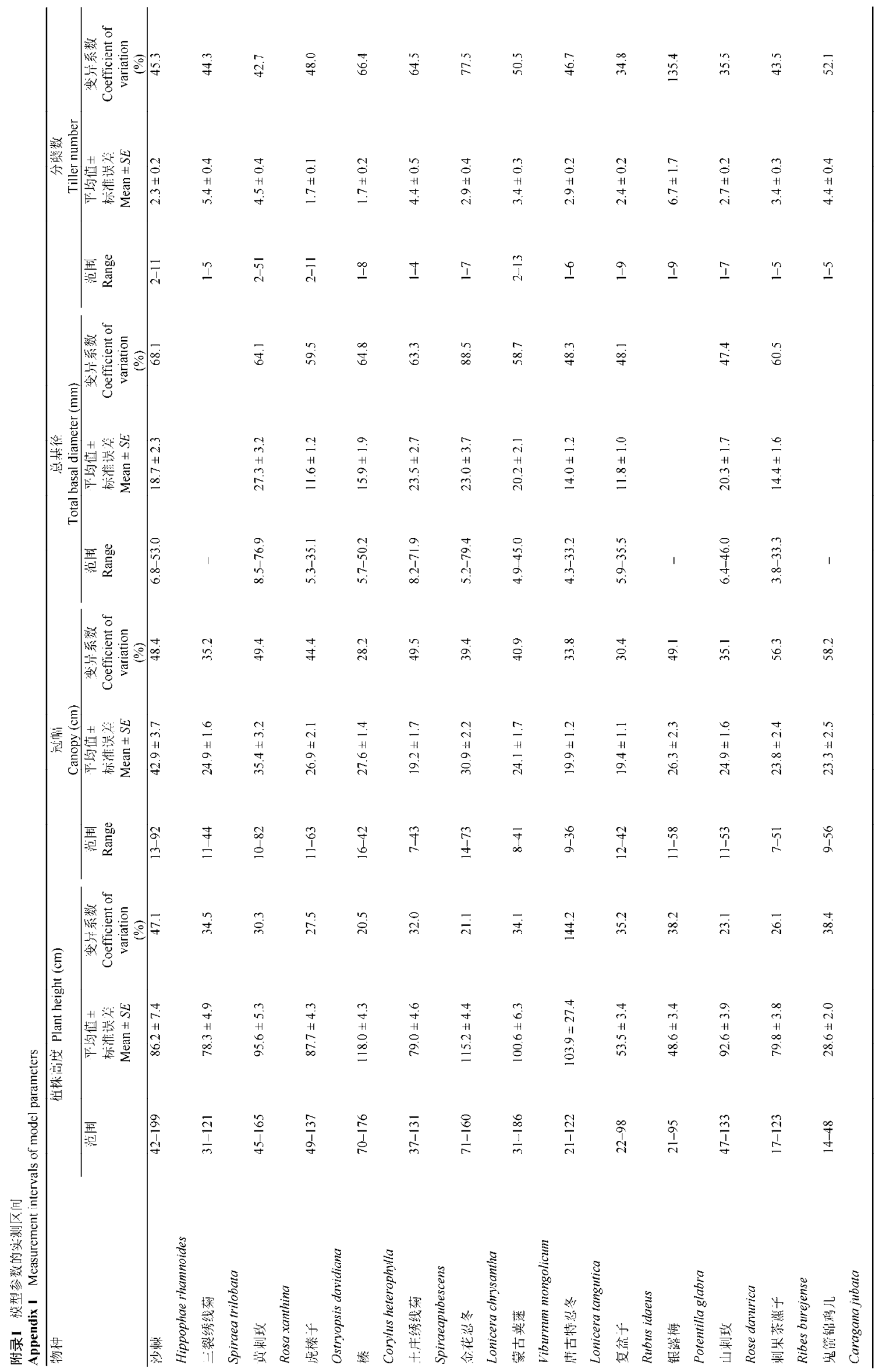




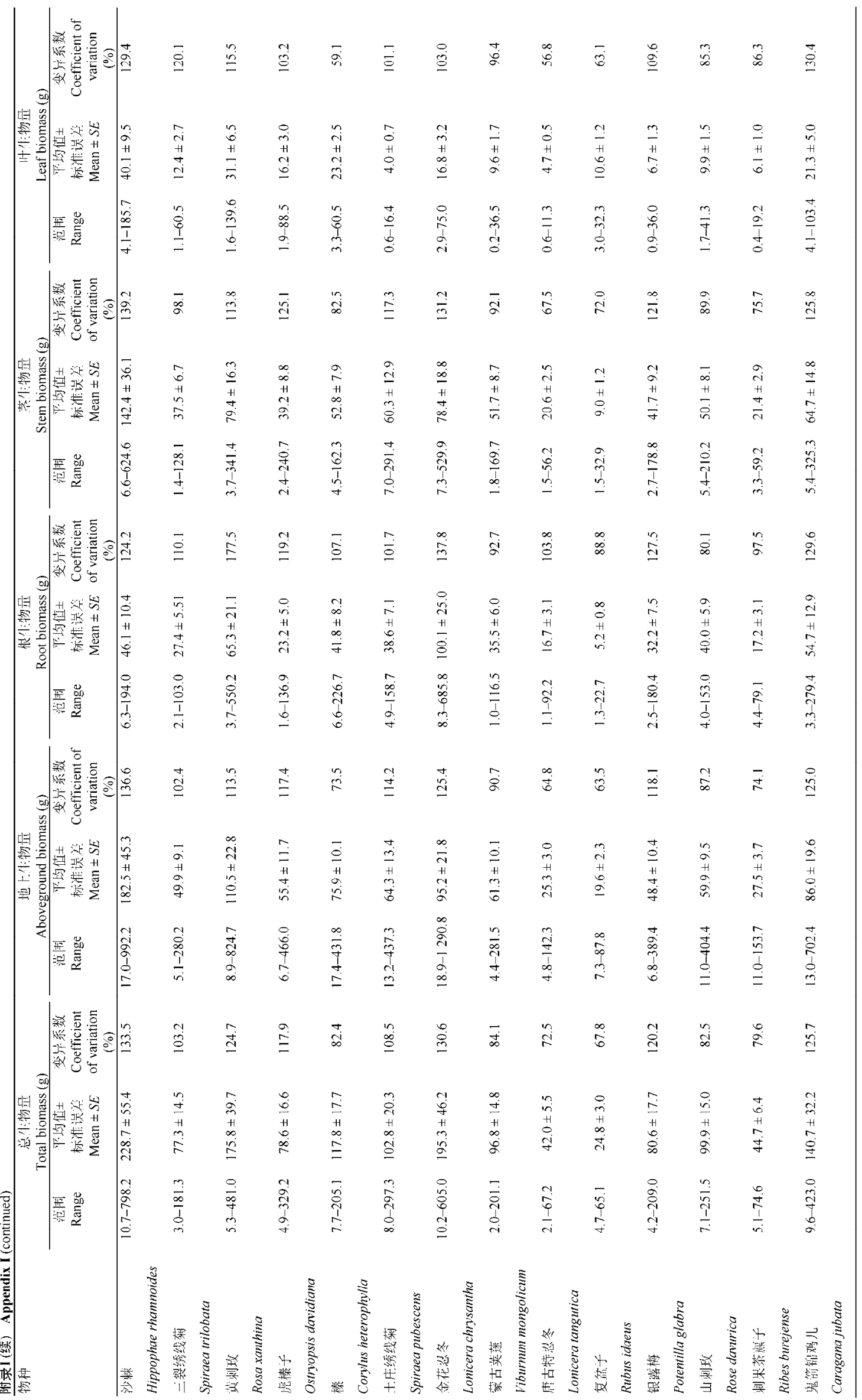

doi: 10.17521/cjpe.2016.0131 


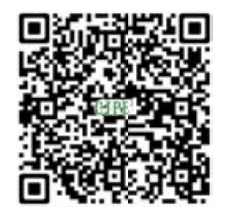

植物生态学报官网

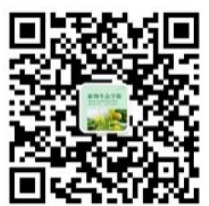

微信订阅号

期刊及学科

相关信息发布

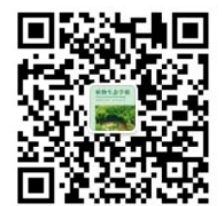

微信服务号

稿件状态查询

全文检索浏览 